\title{
25 Research Soure \\ Remote phosphor-based extended white light source pumped by single blue LED for efficient lighting
}

Atul Kumar Dubey ( $\sim$ atuldubey413@gmail.com )

Indian Institute of Technology Delhi, India https://orcid.org/0000-0002-0568-5729

Shivam Trivedi

Indian Institute of Technology Delhi, India https://orcid.org/0000-0003-3943-6007

Vikas Kumar

Indian Institute of Technology Delhi, India https://orcid.org/0000-0001-5412-2802

Puspita Chanda

Indian Institute of Technology Delhi, India https://orcid.org/0000-0002-5158-7070

Dalip Singh Mehta ( $\nabla$ mehtads@physics.iitd.ac.in )

Indian Institute of Technology Delhi, India https://orcid.org/0000-0002-1569-1647

\section{Research Article}

Keywords: Solid-State Lighting, Remote Phosphor LED, Broadband Illumination

Posted Date: January 17th, 2022

DOI: https://doi.org/10.21203/rs.3.rs-1266211/v1

License: (9) This work is licensed under a Creative Commons Attribution 4.0 International License.

Read Full License 


\section{Abstract}

We report the development of an LED-based extended white light source containing a small cylindrical acrylic diffuser rod coated with YAG: $\mathrm{Ce}^{+3}$ phosphor and excited by a single $1 \mathrm{~W}$ blue LED. This design supports remote phosphor coating technique and thermally isolates both LED as well as the phosphor layer. In this work, the blue light generated from GaN based LED chip was waveguided throughout the cylindrical diffuser and properly excited the phosphor layer for photoluminescence uniformity. The gradient diffusing technique was adopted for optimal light distribution of scattering and waveguiding of blue photons. The process dealt with both specular transmittance and bidirectional transmission distribution function (BTDF) of the diffuser so that the waveguided blue photons could propagate longer distance inside the diffuser rod via total internal reflection mechanism with simultaneous scattering due to gradient diffusing surface structure. The blue photons were found nearer to yellow phosphor behaved like an integrated chip-based system in remote phosphor structure. The scattering of blue photons was used in the photoluminescence process which was uniform throughout the diffuser length due to the selective diffusing technique. Because of this arrangement, the backward fluorescence and backreflection of blue photons to the LED chip from phosphor layer were reduced significantly, resulting in the improvement of the source's luminance properties. The optical properties were properly analyzed, and it was found suitable for the use of general-purpose illumination-based applications.

\section{Introduction}

White light emitting diode (WLED) after the invention of highly efficient GaN blue LED has replaced the conventional light sources like incandescent lamps, compact fluorescence lamp (CFL) and discharge lamps for general illumination purpose [1-3]. LED offers many advantages such as energy saving, longer lifetime, environmental friendliness and instant start than other light sources like CFL, halogen lamps [4]. LEDs play most important part in solid state lighting (SSL) for general lighting and illumination. The main methods for generation of white light using LED are (1) color mixing via combination of red, green and blue (RGB) LEDs [5]; (2) Down-conversion (DC) using UV LED excitation of red, green, blue (RGB) phosphor [6] and (3) DC using blue LED with yellow phosphor (YAG: $\left.\mathrm{Ce}^{+3}\right)[1,7-8]$. All these three processes for white light generation have their own advantages and disadvantages corresponding to their related applications [8-9]. The most promising method for white light generation is with blue LED and YAG: $\mathrm{Ce}^{+3}$ phosphor because of the use of single phosphor layer with single excitation source $[1,8]$. In this combination blue light generated from LED excites the YAG: $\mathrm{Ce}^{+3}$ to emit the yellow light by downconverting the blue component [8-10]. In addition, some of the blue photons still remain unutilized following the direct transmission and scattering process [8-10]. The DC yellow light and residual blue light coming from phosphor layer is mixed to generate a proper white light. The luminous efficacy of this white light source is better than the white light with UV and RGB phosphors because the RGB phosphors as combination are responsible for light quenching [8]. YAG: $\mathrm{Ce}^{+3}$ is an inorganic rare earth material with high quantum efficiency, long life-time and good color parameters [8, 11-12]. It has been reported that YAG: $\mathrm{Ce}^{+3}$ shows drop in emission intensity with the different temperatures generated from non-radiative 
transition of LED [13-16]. Both, the backward fluorescence and the blue photon back-reflection from phosphor layer are responsible for high nonradiative relaxation rates inside the LED junction which leads high temperature generation at LED-phosphor interface [16-19]. On increasing the temperature, the phosphor shows higher luminance and photoluminescence quenching which affects the luminous efficacy and life time of white light source [16-19]. To reduce this heating effect, remote phosphor coating methods were developed but the LED is incoherent with high scattering so phosphor cannot be placed far away from LED to avoid the heating effect [20-22]. In this method, a slight improvement in the luminance was observed but quenching effect was not removed completely or significantly [20-22]. The back reflection of blue component was reduced due to high blue irradiance absorption but backward fluorescence is still an issue for high non-radiative inside the LED junction which affects the life time of high brightness LED [13, 22-24]. Therefore, multiple small blue LEDs (MSB-LED) are being used to replace high brightness LED and it is currently a successful approach for higher efficacy illumination [2526]. In MSB-LEDs system, the problem of backward fluorescence is still a big issue which results in collective heating from each LED junction to the phosphor layer which is luminescent properties of phosphor, discoloration, decohesion, and cracking of the interface layer [26-28]. Further, the multiple blue LEDs lead to nonuniform illumination and multiple bright spots leading to the glare in the eye [26-28]. Therefore, the current requirement for illumination application is to resolve the efficiency droop problem in LED but the solution to this problem is still the topic of debate for the III-nitride LED research community and significant improvement is a hope from decades but not yet achieved [29-31]. The alternate method is remote phosphor method which needs to be improved because there is hazardous spot scattering and backward luminance absorption to LED.

We developed an extended diffuser-based white LED source to make both the phosphor layer and blue LED chip independent of each other for stable illumination. This remote phosphor-based design consists of 1-watt high power blue LED of $457 \mathrm{~nm}$ peak wavelength and a cylindrically shaped diffuser of $30 \mathrm{~mm}$ length and $8 \mathrm{~mm}$ diameter with the phosphor coating onto the circumference surface of cylindrical rod. The diffuser is made up of acrylic rod of refractive index (RI) 1.49, nearly transparent in the visible region similar to glass with $1.52 \mathrm{RI}$. The phosphor layer was the composition of YAG: $\mathrm{Ce}^{+3}$ mixed with UV-visible adhesive (epoxy) with 7(phosphor):2(epoxy) ratio. The acrylic rod was first diffused with a gradient diffusing technique for optimal excitation of the phosphor layer with the help of bidirectional scattering distribution function (BSDF) form lower to higher value from initial to middle of the rod and from higher value to lower value from middle section to final section of the rod. This resulted in proper scattering and waveguiding of blue photons inside and at the surface of the cylindrical diffuser. In this system, the blue light was waveguided inside the diffuser to a sufficiently long distance of $30 \mathrm{~mm}$ compared to other reported remote phosphor-based white LEDs. The phosphor layer was then coated on the acrylic diffuser's surface with a variable concentration in the process of gradient diffusing. Due to the proposed gradual diffusing and varying concentration of phosphor layer, uniformity in the photoluminescence was achieved and the improvement in the luminance uniformity was observed compared to normal diffusing method. To further improve luminance in the forward direction a parabolic reflector cup was used for redirecting the light to the desired area for improving the intensity of the light source in the forward 
direction. The device is useful to support the internal structure of light system such as flood lights, stadium lights, automobile headlights and display based illumination applications. All the optical parameters were properly analyzed and it was foud suitable for illumination applications.

\section{Development Of Led-based Extended White Light Source}

\section{1-Dimensional Analysis of Diffuser for Single LED Excitation}

The two blue LEDs $(\lambda=457 \mathrm{~nm}, \Delta \lambda=25 \mathrm{~nm})$ of 1 -watt optical power each were mounted at the two ends of the acrylic rod and the blue color light was waveguided through a cylindrical rod of $20 \mathrm{~cm}$ and $8 \mathrm{~mm}$ diameter, Fig. 1(a). The cylindrical rod was made of acrylic material with refractive index of 1.49, and it was highly transparent in the visible region above $425 \mathrm{~nm}$. The transmission spectrum of acrylic material is shown in Fig. 1(b) which shows that the transmission of visible light is much higher above 425nm up to near infrared region. Figure 1 (c) shows the photograph of the polished cylindrical acrylic rod with wave guiding and non-uniform scattering of blue light. First the experiment was conducted without diffusing the rod and the phosphor layer of $150 \mu \mathrm{m}$ thickness with a concentration of $0.0050 \mathrm{gram} / \mathrm{cm}^{2}$ was coated using screen printing technique, Fig. 1(d). Without diffusing the rod, most of the blue light was wave-guided through it with some light extraction at the ends. As can be seen from Fig. 1(e), i.e., the yellow phosphor was not excited at the middle of the acrylic rod. The photoluminescence $(\mathrm{PL})$ spectra were recorded along the length of the cylindrical rod and the results are shown in Fig. 1 (f). As can be seen from this figure that due to improper excitation of yellow phosphor by blue photons the PL spectra were non-uniform. The PL spectra were recorded using fiber optic spectrometer. The fiber tip was kept at $5 \mathrm{~mm}$ away from the cylindrical surface for each measurement

In order to improve the scattering of blue light uniformly along the length of the acrylic rod, the gradient diffusing technique was adopted. To accomplish the uniform distribution of blue photons along the length of rod via gradient diffusing, first of all the simulation study was conducted.

\subsection{Simulation and Experimental Results for PL Uniformity 2.2.1 Simulation for PL Uniformity}

The polished acrylic extended surface of $30 \mathrm{~mm}$ length and $8 \mathrm{~mm}$ diameter was considered for simulation. The TracePro simulation software was used for ray tracing of blue photons at the polished acrylic surface, Fig. 2(a). The specular transmission of the acrylic surface was 0.8 (80\%) with $0.15(15 \%)$ losses due to total material absorption or absorption $/ \mathrm{mm}$ and back reflections. It was found that most of the blue components in the middle section of the small rod were waveguided to the other end, Fig. 2(b) and no scattering of blue photons at the center was observed. The simulation results of the polished acrylic extended surface justified the experimental results of PL non-uniformity, Fig. 1(e). Maximum blue photons were waveguided inside the acrylic rod resulting unexcited phosphor layer in the middle section. 
Therefore, it was decided to diffuse the polished surface of the rod uniformly throughout the length to extract out optimal blue component for phosphor excitation.

This resulted in reducing the specular transmission up to $40 \%$ and increasing the bidirectional transmission distribution function (BTDF) to $40 \%$, Fig. 2(c). The simulation results in Fig. 2(d) shows that the blue light was extracted at the middle section of the diffuser significantly making the scattering nearly uniform from one end to the other end of the rod. But there is still higher blue irradiance at the initial section of the rod this is due to the fact that more number of blue photons were extracted out at the beginning. Therefore, the uniform diffusing method was not suitable for the initial section of rod because it reduced the waveguided photons that were transferred to the middle section, leading to higher initial scattering for phosphor excitation.

The maximum photons must waveguide at the initial section to propagate further into the middle section and the final end of the extended cylindrical diffuser. To resolve this problem, variable diffusing of surface was required which was termed as gradient diffusing technique. In the process of gradient diffusing, first the diffuser was virtually divided into ten sections (3mm each), Fig. 3(a), and the diffuser's initial sections were made with maximum specular transmission to reduce the waveguiding loss at a critical angle inside the diffuser.

Section 1 in Fig. 3 (a) was completely polished after that the rod was properly diffused in ascending order from section 2 to 5 . From sections 6 to 10, the descending order diffusing was maintained. The main motive of this gradient diffusing was to use both waveguiding and scattering properties of the diffuser. The specular transmission (ST) and BTDF parameters taken in diffusing are shown in Fig. 3(b). The simulation showed the proper distribution of blue irradiance at the phosphor layer surface; Fig. 3(c). Fig. 3(d) showed the blue photons and fluorescence photon scattering on the cylindrical circumference detection recorded at a $10 \mathrm{~mm}$ perpendicular distance from the source. Through the simulation parameters of gradient diffusing, the experimental rod was prepared for further development.

\subsubsection{Development of Extended Diffuser for PL Uniformity}

In the development process, the three different small rods (polished, uniform diffusing, and gradient diffusing) were used in accordance with simulation for experimental verification. Fig. 4 (a, b, c) are the schematics of polished, uniform diffusing, and gradient diffusing rods, respectively. Fig. 4 (d) was the spectra of polished rod taken at $5 \mathrm{~mm}$ perpendicular distance from the rod which shows the high nonuniformity in PL of blue radiance along the length of the rod. Fig. 4(e) is the blue irradiance spectra along the length which showed the maximum scattering at initial section of the rod, and there is a large gap between $0 \mathrm{~cm}$ and $2.5 \mathrm{~cm}$ length.

To fill this spectral bridge, the gradient diffusing technique was adopted according to simulation parameters. The spectral gap was observed, Fig. 4(f), but it shows a better result than the previous design because the blue radiance distribution was observed from $0.5 \mathrm{~cm}$ to $2.5 \mathrm{~cm}$, Fig. 4 (d \& e). This was the maximum that can be achieved experimentally through the gradient diffusing method. Considering these 
improvements in blue photon extraction to the phosphor layer, the gradient phosphor coating technique was adopted using the screen-printing technique. In this phosphor coating process, the optimal concentration was used for particular blue irradiance to maintain the PL uniformity, i.e., for higher blue irradiance, the higher concentration of phosphor was adopted, and for lower blue radiance, the required amount of phosphor concentration was adopted for same PL. The same thickness of the phosphor layer was maintained throughout the rod which was $150 \mu \mathrm{m}$, and the phosphor: adhesive ratio was changed along the length, as shown in Fig. 5(a). The total (phosphor + adhesive concentration) was 0.00611 $\mathrm{gm} / \mathrm{cm}^{2}$ throughout the diffuser surface. Fig. 5 (a) shows that adhesive concentration was increased on decreasing the phosphor concentration. The UV adhesive was adopted for phosphor coating because its refractive index is nearer to glass.

As the phosphor concentration + adhesive concentration was managed constant which was 0.00611 $\mathrm{gm} / \mathrm{cm}^{2}$, the layer thickness was $150 \mu \mathrm{m}$ throughout the length which simplified the design. The variation of phosphor and adhesive concentration (7:2) also indicated that the phosphor layer's refractive index was continually changing from high value to lower value, resulting in higher extraction of blue source along the length of the diffuser to adjust the maximum illuminance uniformity, Fig. 5(b). The 3D design is shown in Fig. 6(a) of phosphor layer coating on diffusers' surface from higher to lower value. When the blue irradiance from a single LED source was properly distributed inside the extended diffuser, the approximate PL uniformity was observed, as shown in Fig. 6(b). The lighting device illuminated the same color along each section of the diffuser due to PL uniformity. This work can be internally involved in illumination systems such as automobile LED headlight structures. The intensity along the length of rod with gradient diffusing (GD) was improved in the term of illuminance uniformity than the uniform diffusing (UD) technique-based diffuser, Fig. 6(c). But the intensity at the diffuser's initial sections was still higher because of higher scattering from the blue LED.

In contrast, the dome-shaped structure in the diffuser for the LED system helped in the directionality, but it was not enough.

To normalize the higher luminance observed at the initial section, the diffuser was covered by a parabolic reflector to redirect the light towards the desired region which is shown in figure 7(a). Figure 7(b) shows the photograph of the source without the mirror at the end section.

The light was uniform and less hazardous in comparison to chip-on-board LED or surface-mounted LED. The spectrum was taken along the length of the rod without mirror coating. This was to understand the exact waveguiding of the blue component throughout the rod after the gradient diffusing technique. The light was observed approximately uniform. After mirror coating at the end of the rod, enhancement in light was improved for non-hazardous and efficient illumination.

\section{Results And Discussion}


The proposed pc-LED remote phosphor-based extended white light source associated the color coordinates of $(0.32,0.33)$, correlated color temperature (CCT) of $6300{ }^{\circ} \mathrm{K}$, and color rendering index (CRI) of 79 recorded by Lisun-6000 spectrometer as shown in Fig. 8 (a, b, c, d). The obtained color co-ordinates were quite close to an ideal white light source having $(0.33,0.33)$. The slight difference from the ideal values is due to dip in the spectral profile in green region and lesser amount of red color photons.

The CCT of $6300{ }^{0} \mathrm{~K}$ is a cool white this is due to missing deep red components. For an ideal white light source, the CRI value is supposed to more than 95 , however in our case it less than 90 the same is also attributed to missing green color and deep red color photons. Nevertheless, the color parameters were good and acceptable in reference to general illumination. This developed light source was cool white light. To improve CRI above 85, the red phosphor can be used for increasing the R9 value, Fig. 8 (c), but this comes with the cost of intensity loss due to self-absorption between the phosphors. The PL uniformity was achieved by controlling the blue photons using the gradient diffusing technique so that the blue photons may properly follow the total internal reflection as well as scattering at the diffuser surface. There are mainly two advantages of waveguiding the blue photons inside the diffuser; (1) this source is a remote phosphor-based system, but the blue photons are much nearer to phosphor same as the integrated pc-LED system which fulfills the requirement of many LED; (2) both LED and phosphor are independent to each other for stable photoluminescence and minimal back-reflection losses responsible for lesser self-absorption in LED.

The blue component of the light source was waveguided at a $3 \mathrm{~cm}$ long distance inside the acrylic diffuser which separated phosphor away from the LED chip to avoid heating effect on the phosphor. The $8 \mathrm{~mm}$ was the most appropriate diameter where we can control the blue photons for uniform white light illumination, and below this, high precision was required in diffusing. Above $8 \mathrm{~mm}$, the length should be decreased to avoid high diffusing blue light losses inside the rod. The luminous efficacy of our LEDbased extended white light source was $70 \mathrm{~lm} /$ watt. Although 1-watt optical blue LEDs are considered to have 90 lumens, the losses are phosphor absorption, acrylic absorption/mm, and self-absorption inside the system. The acrylic diffuser separated the phosphor layer from the blue LED chip; therefore, the backward fluorescence and back reflection reduced the rate of nonradiative relaxation inside the LED junction due to the phosphor layer. The back-scattered blue photons were used again in the fluorescence conversion process due to the total internal reflections inside the diffuser rod which is the advantage of this device. The proposed remote phosphor-based method for efficient white light generation is useful and may be used in the internal and external lighting system design such as automobile headlight systems and phosphor converted LED based displays.

\section{Conclusion}

We have developed a remote phosphor-coated extended white light source using YAG: $\mathrm{Ce}^{+3}$ pumped by single 1-watt blue LED. In this proposed design, scattered blue light from LED was made to waveguide through a diffuser and uniformly extracted out due to selective BSDF property applied on cylindrical surface during the diffusing process. This cylindrical acrylic diffuser was developed with a gradient 
diffusing technique. YAG: $\mathrm{Ce}^{+3}$ phosphor was coated in the process of gradient diffusing for total internal reflection to avoid the mismatch of scattering for PL uniformity. A blue light component was uniformly dispersed and extracted out from each diffuser section and excited the phosphor layer. The phosphor layer was also coated with varying concentration along the length of the diffuser rod such that uniform $\mathrm{PL}$ is maintained. This yellow light from the phosphor and original blue light was combined together was used to generate white light. The intensity of the light source was improved by using a parabolic cup reflector. The advantage of using an acrylic diffuser was to waveguide the blue component uniformly and avoided the back reflection loss in a remote phosphor-based white LED system to protect the LED chip and phosphor from the heating effect. This kind of design is the replacement of multiple LEDs with a single LED to improve the system's luminous properties. The designed source was less hazardous to the human eye and vision because of uniform illumination of the source, and no bright spots were visible as they are visible in other high-powered white LED systems having multiple blue color chips.

\section{Declarations}

Funding. DST Government of India, DST/TMD/CERI/C24(G)

Conflict of Interest: Authors declare no conflict of interest.

Acknowledgement. Portions of this work were presented at the conference "Light, Energy and Environment 2018" in year 2018 "LED-based extended white light source to reduce the heating effect on YAG: $\mathrm{Ce}^{+3}$ phosphor". ST5D.3. Authors are thankful to Mayank Gupta and Virendra Kumar, Bio \& Green Photonics Laboratory, Department of Physics, IIT Delhi, for their valuable help and support.

\section{References}

1. S. Nakamura, The invention of high efficient blue LEDs and future Solid State lighting, in: Light. Energy Environ., Optical Society of America, Leipzig, 2016: p. JM1A.1. https://doi.org/10.1364/FTS.2016.JM1A.1

2. V.K. Khanna, Fundamental of solid state lighting LED,OLED and Their Applications in Illumination and Displays, CRC Press, Taylor \& Francis Group, Florida, United States, 2014.

3. M.H. Crawford, J.J. Wierer, A.J. Fischer, G.T. Wang, D.D. Koleske, G.S. Subramania, M.E. Coltrin, R.F. Karlicek, J.Y. Tsao, Solid-state lighting: Towards smart and ultraefficient materialacs, device, lamps and systems, in: D.L. Andrews (Ed.), Photonics Photonics Technol. Instrum., Wiley, New York, 2015: pp. 1-56. https://doi.org/10.1002/9781119011781.

4. X. Luo, R. Hu, S. Liu, K. Wang, Heat and fluid flow in high-power LED packaging and applications, Prog. Energy Combust. Sci. 56 (2016) 1-32. https://doi.org/https://doi.org/10.1016/j.pecs.2016.05.003. 
5. S. Muthu, F.J.P. Schuurmans, M.D. Pashley, Red, green, and blue LEDs for white light illumination, IEEE J. Sel. Top. Quantum Electron. 8 (2002) 333-338. https:// doi.org/10.1109/2944.999188.

6. Y. Muramoto, M. Kimura, A. Dempo, S. Nouda, Y. Fukawa, S. Sakai, High-efficiency UV LEDs and RGB white LEDs for lighting and LCD backlights, J. Soc. Inf. Disp. 19 (2011) 907. https://doi.org/10.1889/jsid19.12.907.

7. Y.-C. Lin, M. Karlsson, M. Bettinelli, Inorganic Phosphor Materials for Lighting, Top. Curr. Chem. 374 (2016) 21. https://doi.org/10.1007/s41061-016-0023-5.

8. A.A. Setlur, Phosphors for LED- based Solid - State Lighting, Electrochem. Soc. Interface. 18 (2009) 32-36. https://www.electrochem.org/dl/interface/wtr/wtr09/wtr09_p032-036.pdf

9. L. Chen, C.C. Lin, C.W. Yeh, R.S. Liu, Light converting inorganic phosphors for white light-emitting diodes, Materials (Basel). 3 (2010) 2172-2195. https://doi.org/10.3390/ma3032172.

10. A. Zabiliûtë, R. Vaicekauskas, P. Vitta, A. Žukauskas, Phosphor-converted leds with low circadian action for outdoor lighting, Opt. Lett. 39 (2014) 563-566. https://doi.org/10.1364/OL.39.000563.

11. H. Shi, C. Zhu, J. Huang, J. Chen, D. Chen, W. Wang, F. Wang, Y. Cao, X. Yuan, Luminescence properties of YAG:Ce, Gd phosphors synthesized under vacuum condition and their white LED performances, Opt. Mater. Express. 4 (2014) 649. https://doi.org/10.1364/OME.4.000649.

12. S. Nishiura, S. Tanabe, K. Fujioka, Y. Fujimoto, Properties of transparent Ce:YAG ceramic phosphors for white LED, Opt. Mater. (Amst). 33 (2011) 688-691.

https://doi.org/10.1016/j.optmat.2010.06.005.

13. C.M. Tan, P. Singh, W. Zhao, H.C. Kuo, Physical Limitations of Phosphor layer thickness and concentration for White LEDs, Sci. Rep. 8 (2018) 2452. https://doi.org/10.1038/s41598-018-20883-3.

14. C.C. Sun, Y.Y. Chang, T.H. Yang, T.Y. Chung, C.C. Chen, T.X. Lee, D.R. Li, C.Y. Lu, Z.Y. Ting, B. Glorieux, Y.C. Chen, K.Y. Lai, C.Y. Liu, Packaging efficiency in phosphor-converted white LEDs and its impact to the limit of luminous efficacy, Drug Saf. 1 (2014) 1-17. https://doi.org/10.1186/s40539-014-0019-0.

15. L.L. ZHANG Yanfang, Temperature effects on photoluminescence of YAG:Ce3+ phosphor and performance in white light-emitting diodes, J. Rare Earths. 26 (2008) 446-449. https://doi.org/10.1016/S1002-0721(08)60115-5.

16. Yuan-Chih Lin, Marco Bettinelli, S. K. Sharma, Britta Redlich, Adolfo Speghini, Maths Karlsson, Unraveling the impact of different thermal quenching routes on the luminescence efficiency of the $Y$ 3 Al 50 12:Ce 3+ phosphor for white light emitting diodes, J. Mater. Chem. C. 8 (2020) 1401514027. https://doi.org/10.1039/D0TC03821K.

17. S. Zhou, B. Cao, S. Yuan, S. Liu, Enhanced luminous efficiency of phosphor-converted LEDs by using back reflector to increase reflectivity for yellow light, Appl. Opt. 53 (2014) 8104. https://doi.org/10.1364/ao.53.008104.

18. P. Gorrotxategi, M. Consonni, A. Gasse, Optical efficiency characterization of LED phosphors using a double integrating sphere system, J. Solid State Light. 2 (2015) 1. https://doi.org/10.1186/s40539014-0020-7. 
19. M.A. van de Haar, M. Tachikirt, A.C. Berends, M.R. Krames, A. Meijerink, F.T. Rabouw, Saturation Mechanisms in Common LED Phosphors, ACS Photonics. 8 (2021) 1784-1793. https://doi.org/10.1021/ACSPHOTONICS.1C00372.

20. G. Singh, D.S. Mehta, White-light generation using a remote-phosphor-coated diffusing surface excited by the high-brightness blue light-emitting diode, J. Inf. Disp. 15 (2014) 91-98.

21. Y.H. Chen, N.Z. Zhuo, P. Jiang, S.W. Cheng, P.F. Shen, H.B. Wang, Optical Model of Laminated Remote Phosphor Films and Its Application in White LED, Adv. Condens. Matter Phys. 2019 (2019). https://doi.org/10.1155/2019/1493286.

22. S.H. Abdullayeva, T.Y. Orujov, N.N. Musayeva, R.B. Jabbarov, S.K. Orujov, S.H. Abdullayeva, T.Y. Orujov, N.N. Musayeva, R.B. Jabbarov, S.K. Orujov, Remote Pyramid-Shaped Phosphor Coating for Phosphor-Converted White LEDs, World J. Nano Sci. Eng. 7 (2017) 17-24. https://doi.org/10.4236/WJNSE.2017.72002.

23. H.C. Chen, K.J. Chen, C.H. Wang, C.C. Lin, C.C. Yeh, H.H. Tsai, M.H. Shih, H.C. Kuo, T.C. Lu, A novel randomly textured phosphor structure for highly efficient white light-emitting diodes, Nanoscale Res. Lett. 7 (2012) 1-5. https://doi.org/10.1186/1556-276X-7-188.

24. J.K. Kim, H. Luo, E.F. Schubert, J. Cho, C. Sone, Y. Park, Strongly enhanced phosphor efficiency in GalnN white light-emitting diodes using remote phosphor configuration and diffuse reflector cup, Japanese J. Appl. Physics, Part 2 Lett. 44 (2005). https://doi.org/10.1143/JJAP.44.L649.

25. I. Moreno, Creating a desired lighting pattern with an LED array, in: I.T. Ferguson, T. Taguchi, I.E. Ashdown, S.-J. Park (Eds.), Eighth Int. Conf. Solid State Light., SPIE, 2008: p. 705811. https://doi.org/10.1117/12.795673.

26. H. Zheng, L. Li, X. Lei, X. Yu, S. Liu, X. Luo, Optical performance enhancement for chip-on-board packaging LEDs by adding TiO2/silicone encapsulation layer, IEEE Electron Device Lett. 35 (2014) 1046-1048. https://doi.org/10.1109/LED.2014.2349951.

27. I. Moreno, M. Avendaño-Alejo, R.I. Tzonchev, Designing light-emitting diode arrays for uniform nearfield irradiance, Appl. Opt. 45 (2006) 2265-2272. https://doi.org/10.1364/A0.45.002265.

28. J.K. Sim, K. Ashok, Y.H. Ra, H.C. Im, B.J. Baek, C.R. Lee, Characteristic enhancement of white LED lamp using low temperature co-fired ceramic-chip on board package, Curr. Appl. Phys. 12 (2012) 494-498. https://doi.org/10.1016/j.cap.2011.08.008.

29. X. Luo, X. Fu, F. Chen, H. Zheng, Phosphor self-heating in phosphor converted light emitting diode packaging, Int. J. Heat Mass Transf. 58 (2013) 276-281.

30. J.J. Wierer, J.Y. Tsao, D.S. Sizov, Comparison between blue lasers and light-emitting diodes for future solid-state lighting, Laser Photon. Rev. 7 (2013) 963-993. https://doi.org/10.1002/lpor.201300048.

31. J. Cho, E.F. Schubert, J.K. Kim, Efficiency droop in light-emitting diodes: Challenges and countermeasures, Laser Photon. Rev. 7 (2013) 408-421. https://doi.org/10.1002/Ipor.201200025

\section{Figures}




\section{Figure 1}

(a) Schematic diagram of the polished acrylic rod $20 \mathrm{~cm}$ long with blue LEDs mounted at both the ends, (b) transmission spectrum of acrylic material, (c) scattering of blue light along the $20 \mathrm{~cm}$ polished cylindrical rod, (d) schematic diagram of YAG: $\mathrm{Ce}^{+3}$ coated rod, (e) photograph of the phosphor excited rod using blue LEDs and (f) PL spectra.

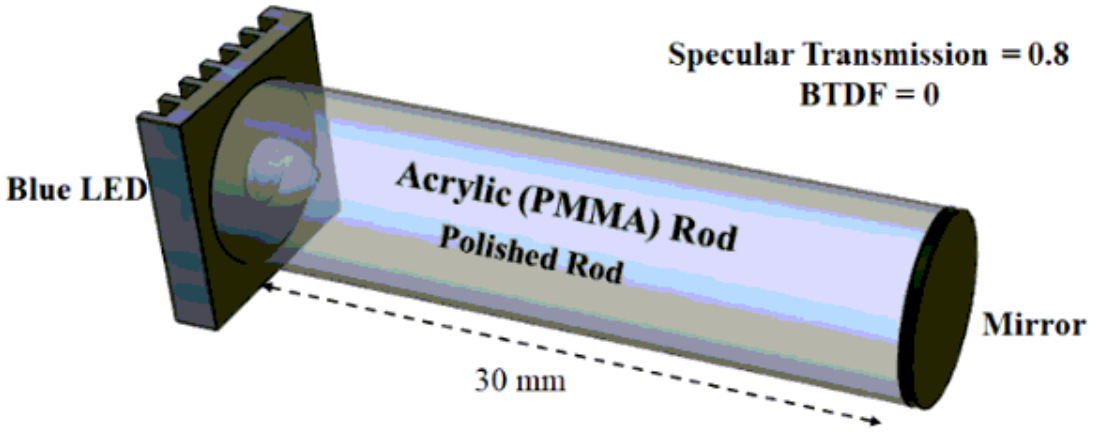

(a)
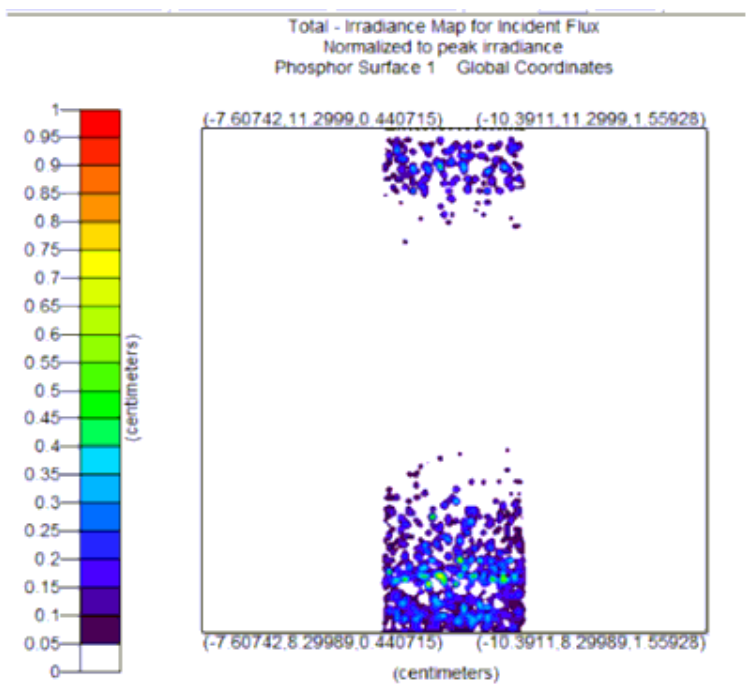

Min 0. Max 1. Ave 0020269

Total Flux 1.5096e-006 W. Fluv/Emitted Flux 0.67094, 2161 incident Rays

(b)
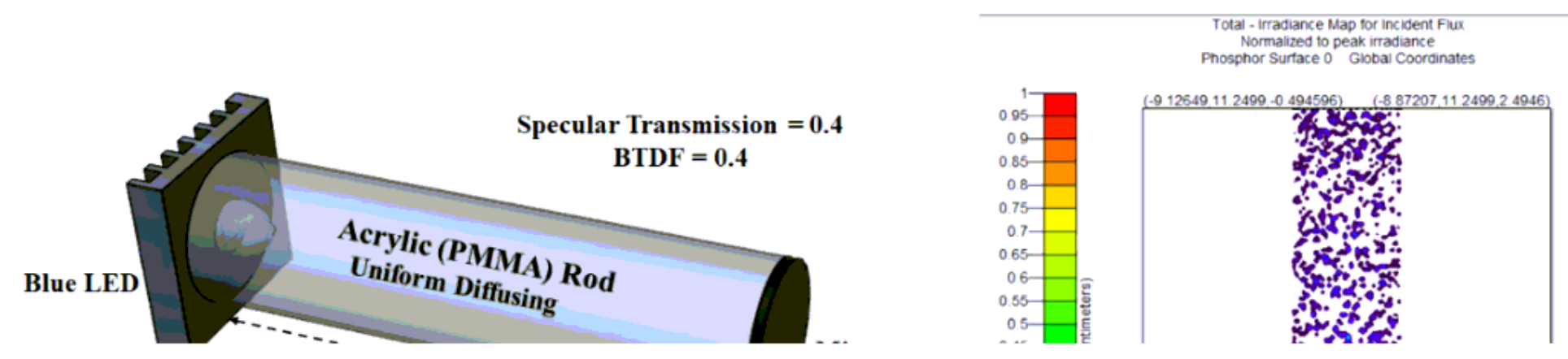

\section{Figure 2}

(a) Schematic of polished acrylic rod (b) simulation of blue irradiance inside the polished acrylic rod; (c) Schematic of uniformly diffused acrylic rod, and (d) simulation of blue irradiance inside the uniformly 


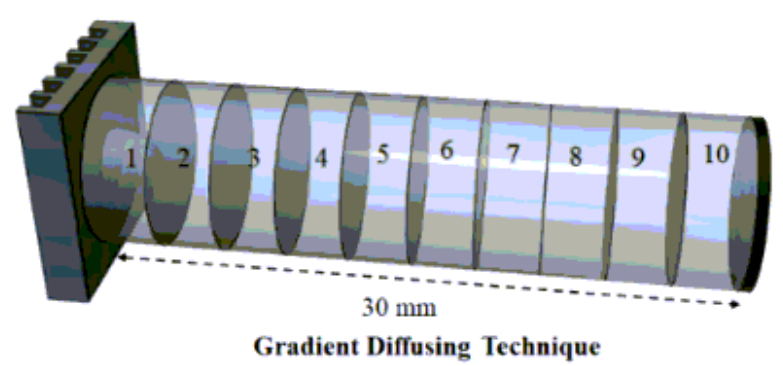

(a)

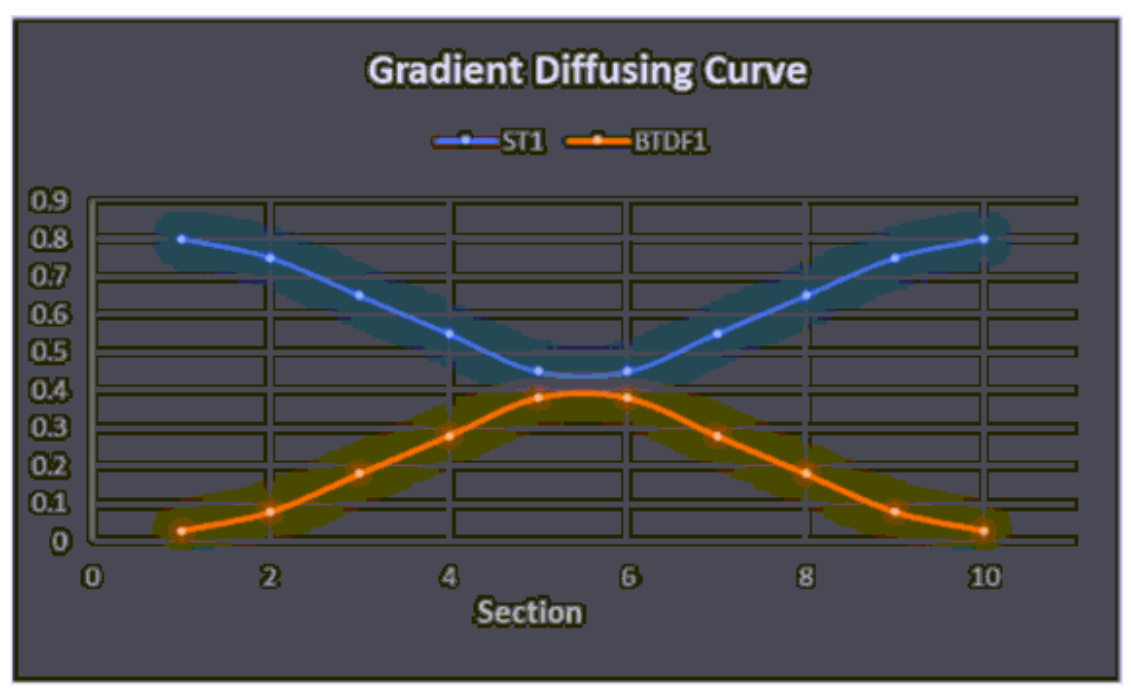

(b)

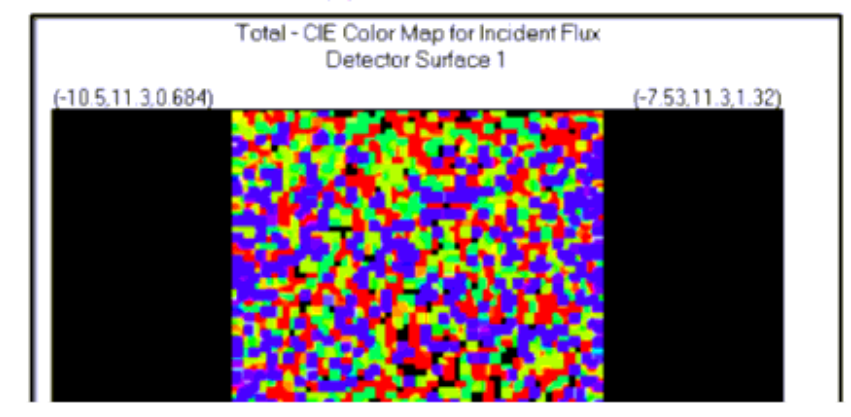

\section{Figure 3}

(a) Schematic diagram of gradually diffused acrylic rod, (b) specular transmission (ST) and bidirectional transmission distribution function (BTDF) for gradient diffusing, (c) simulation of blue irradiance inside the gradient diffusing acrylic rod, and (d) photoluminescence colour map along the length of the diffused rod. 


\section{Figure 4}

Ray optics diagram of light extraction from acrylic rod; (a) polished, (b) uniformly diffused, and (c) gradient diffusing rod, and spectra of scattered blue light recorded along different lengths of rod (d) polished, (e) uniformly diffused, and (d) gradient diffusing rod.

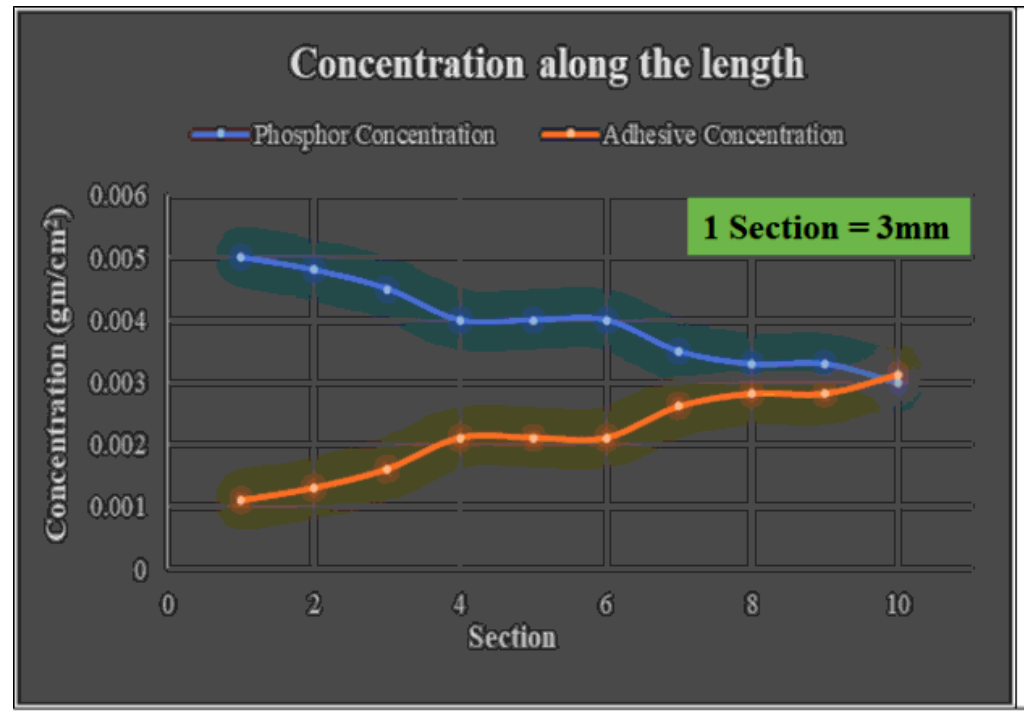

(a)

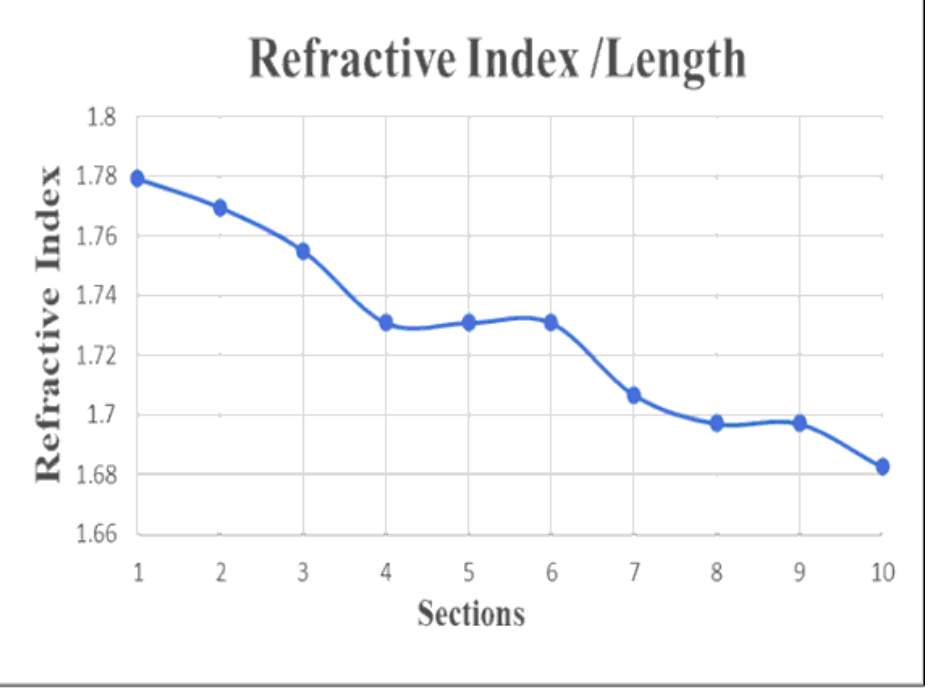

(b)

\section{Figure 5}

(a) Variation of phosphor and adhesive concentration along the length of each section of diffuser rod (thickness og phosphor layer $150 \mu \mathrm{m}$ ), (b) change in the refractive index along the length due to concentration variation. 


\section{Phosphor Coated Diffuser}

(a)

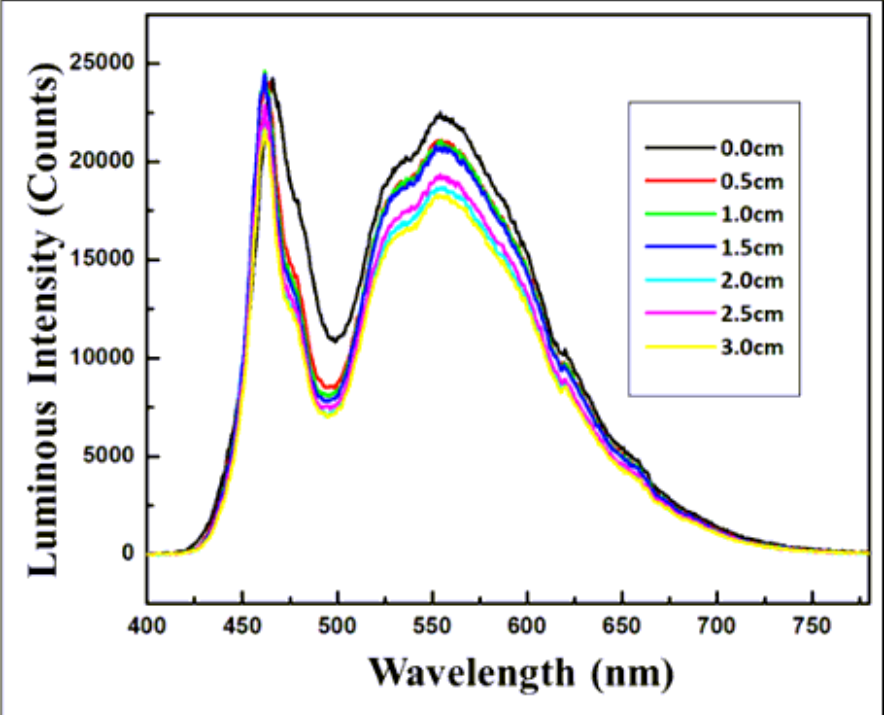

(b)

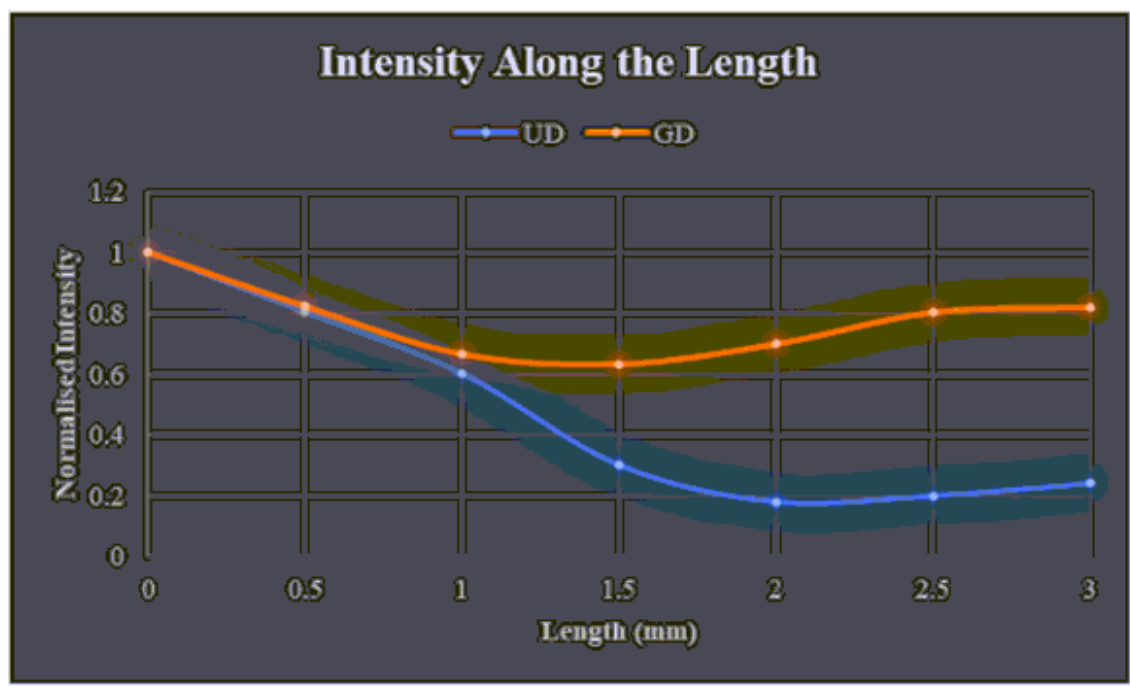

(c)

Figure 6

(a) Schematic diagram of gradient diffusing rod with varying concentration of phosphor, (b) PL spectra recorded at each section of diffuser rod, and (c) compression between the illuminance uniformity for both uniform diffusing (UD) and Gradient diffusing (GD) rod. 

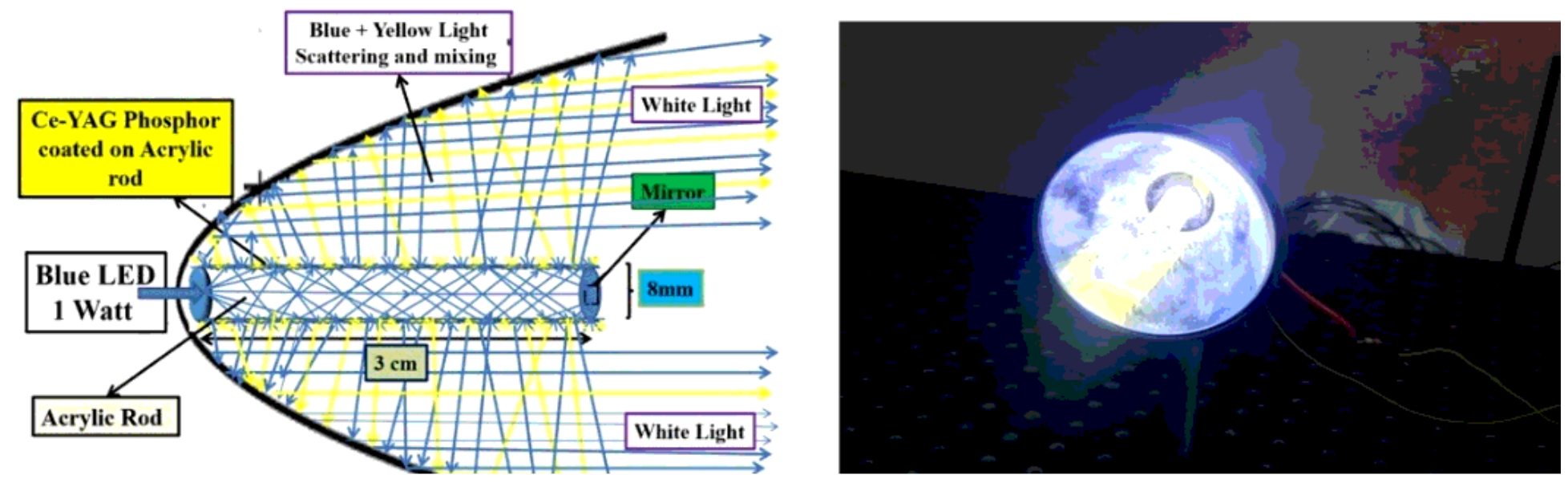

\section{Figure 7}

(a) Schematic diagram of the developed white light source with parabolic cup reflector, (b) photograph of the source without mirror coating at the final end of the gradient diffuser.

\section{Figure 8}

(a) Photograph of developed light source with uniform illumination, (b) Spectrum of the light source recorded at $15 \mathrm{~cm}$ away from the source, (c) CIE 1931 (x, y) colour co-ordinates diagram of the source, and (d) color rendering index (Ra) chart of the source (R1-R15) CRI. 